\title{
Statistical Analysis of Exponential Lifetimes under an Adaptive Type-II Progressive Censoring Scheme
}

\author{
H. K. T. Ng*, D. Kundu', P. S. Chan**
}

\begin{abstract}
In this paper a mixture of Type-I censoring and Type-II progressive censoring schemes, called an adaptive Type-II progressive censoring scheme, is introduced for life testing or reliability experiments. For this censoring scheme, the effective sample size $m$ is fixed in advance and the progressive censoring scheme is provided but the number of items progressively removed from the experiment upon failure may change during the experiment. If the experimental time exceeds a prefixed time $T$ but the number of observed failures does not reach $m$, we terminate the experiment as soon as possible by adjusting the number of items progressively removed from the experiment upon failure. Computational formulae for the expected total test time are provided. Point and interval estimation of the failure rate for exponentially distributed failure times are discussed for this censoring scheme. The various methods are compared using Monte Carlo simulation.
\end{abstract}

Keywords: Life testing; maximum likelihood estimator; asymptotic methods; coverage probability; exponential distribution.

Postal address: *Department of Statistical Science, Southern Methodist University, 3225 Daniel Avenue, Dallas, Texas, USA 75275-0332

Postal address: ${ }^{\dagger}$ Department of Mathematics and Statistics, Indian Institute of Technology, Kanpur 208016, India.

Postal address: ** Department of Statistics, The Chinese University of Hong Kong, Shatin, Hong Kong 


\section{Introduction}

In life testing and reliability studies, the experimenter may not always obtain complete information on failure times for all experimental units. Data obtained from such experiments are called censored data. Reducing the total test time and the associated cost is one of the major reasons for censoring. A censoring scheme, which can balance between (i) total time spent for the experiment; (ii) number of units used in the experiment; and (iii) the efficiency of statistical inference based on the results of the experiment, is desirable.

The most common censoring schemes are Type-I (time) censoring, where the life testing experiment will be terminated at a prescribed time $T$, and Type-II (failure) censoring, where the life testing experiment will be terminated upon the $r$-th ( $r$ is pre-fixed) failure. However, the conventional Type-I and Type-II censoring schemes do not have the flexibility of allowing removal of units at points other than the terminal point of the experiment. Because of this lack of flexibility, a more general censoring scheme called progressive Type-II right censoring has been introduced. Briefly, it can be described as follows: Consider an experiment in which $n$ units are placed on a life testing experiment. At the time of the first failure, $R_{1}$ units are randomly removed from the remaining $n-1$ surviving units. Similarly, at the time of the second failure, $R_{2}$ units from the remaining $n-2-R_{1}$ units are randomly removed. The test continues until the $m$-th failure at which time, all the remaining $R_{m}=n-m-R_{1}-R_{2}-\cdots-R_{m-1}$ units are removed. The $R_{i}^{\prime} s$ are fixed prior to the study. Readers may refer to Balakrishnan [1] and Balakrishnan and Aggarwala [2] for extensive reviews of the literature on progressive censoring.

Recently, Kundu and Joarder [16] proposed a censoring scheme called Type-II progressive hybrid censoring scheme, in which a life testing experiment with progressive Type-II right censoring scheme $\left(R_{1}, R_{2}, \ldots, R_{m}\right)$ is terminated at a prefixed time $T$. However, the drawback of the Type-II progressive hybrid censoring, similar to the conventional Type-I censoring (time censoring), is that the effective sample size is random and it can turn out to be a very small number (even equal to zero), and therefore the standard statistical inference procedures may not be applicable or they will have low efficiency. In this paper we suggest an adaptive Type-II 
progressive censoring, where we allow $R_{1}, R_{2}, \ldots, R_{m}$ to depend on the failure times so that the effective sample size is always $m$, which is fixed in advance. A properly planned adaptive progressively censored life testing experiment can save both the total test time and the cost induced by failure of the units and increase the efficiency of statistical analysis.

The rest of the paper is organized as follows. In Section 2, we first introduce the notation and describe the adaptive Type-II progressive censoring scheme. In Section 3, when the underlying lifetime distribution is exponential, we derive the MLE of the failure rate and discuss the construction of confidence intervals for the failure rate by different methods. Section 4 provides the computation formulae for the expected total test time which will be useful for experimental planning purposes. In Section 5, the efficiency of the MLEs based on the proposed censoring scheme with the Type-II progressive hybrid censoring scheme proposed by Kundu and Joarder [16] is compared. Confidence intervals obtained by different methods are also compared in terms of their coverage probabilities and expected widths by means of extensive Monte Carlo simulations.

\section{Model Description}

Suppose $n$ units are placed on a life testing experiment and let $X_{1}, X_{2}, \ldots, X_{n}$ be their corresponding lifetimes. We assume that $X_{i}, i=1,2, \ldots, n$ are independent and identically distributed with probability density function (PDF) $f_{X}(x ; \boldsymbol{\theta})$ and cumulative distribution function $(\mathrm{CDF}) F_{X}(x ; \boldsymbol{\theta})$, where $\boldsymbol{\theta}$ denotes the vector of parameters and $x \in[0, \infty)$. Prior to the experiment, an integer $m<n$ is determined and the progressive Type-II censoring scheme $\left(R_{1}, R_{2}, \ldots, R_{m}\right)$ with $R_{i}>0$ and $\sum_{i=1}^{m} R_{i}+m=n$ is specified. During the experiment, the $i$-th failure is observed and immediately after the failure, $R_{i}$ functioning items are randomly removed from the test. We denote the $m$ completely observed (ordered) lifetimes by $X_{i: m: n}^{\left(R_{1}, R_{2}, \ldots, R_{m}\right)}, i=1,2, \ldots, m$, which are the observed progressively Type-II right censored sample. For convenience, we will suppress the censoring scheme in the notation of the $X_{i: m: n}$ 's. We also denote the observed values of such a progressively Type-II right censored sample by $x_{1: m: n}<x_{2: m: n}<\cdots<x_{m: m: n}$. 
As noted by Burkschat [7] and Ng, Chan and Balakrishnan [20], it is expected that a progressive censoring plan has a longer test duration than a single (conventional Type-II) censoring plan in return for the gain in efficiency. The value of $R_{i}$ at the time of the $i$-th failure $X_{i: m: n}$ may be determined depending on the objective of the experimenter. The objective may be controlling the total test time or having a higher chance to observe some large failure times (usually leading to a gain in efficiency for statistical inference). Suppose the objective is to control the total test time, a reasonable design to control the total test time is to terminate the experiment at a prefixed time. This problem is considered in [16] for a fixed progressive censoring scheme $\left(R_{1}, R_{2}, \ldots, R_{m}\right)$ and they called this type of censoring Type-II progressive hybrid censoring. The drawback of this censoring scheme is that the effective sample size is random and it can turn out to be a very small number (even equal to zero) so that usual statistical inference procedures will not be applicable or they will have low efficiency. Therefore, we suggest an adaptive censoring scheme in which the effective sample size $m$ is fixed in advance and the progressive censoring scheme $\left(R_{1}, R_{2}, \ldots, R_{m}\right)$ is provided, but the values of some of the $R_{i}$ may change accordingly during the experiment.

Suppose the experimenter provides a time $T$, which is an ideal total test time, but we may allow the experiment to run over time $T$. If the $m$-th progressively censored observed failure occurs before time $T$ (i.e. $X_{m: m: n}<T$ ), the experiment stops at the time $X_{m: m: n}$ (see Figure 1(a)). Otherwise, once the experimental time passes time $T$ but the number of observed failures has not reached $m$, we would want to terminate the experiment as soon as possible. This setting can be viewed as a design in which we are assured of getting $m$ observed failure times for efficiency of statistical inference and at the same time the total test time will not be too far away from the ideal time $T$. From the basic properties of order statistics (see, for example, David and Nagaraja [10], Section 4.4), we know that the fewer operating items are withdrawn (i.e., the larger the number of items on the test), the smaller the expected total test time (Ng and Chan [19]). Therefore, if we want to terminate the experiment as soon as possible for fixed value of $m$, then we should leave as many surviving items on the test as possible.

Suppose $J$ is the number of failures observed before time $T$, i.e.

$$
X_{J: m: n}<T<X_{J+1: m: n}, \quad J=0,1, \ldots, m,
$$




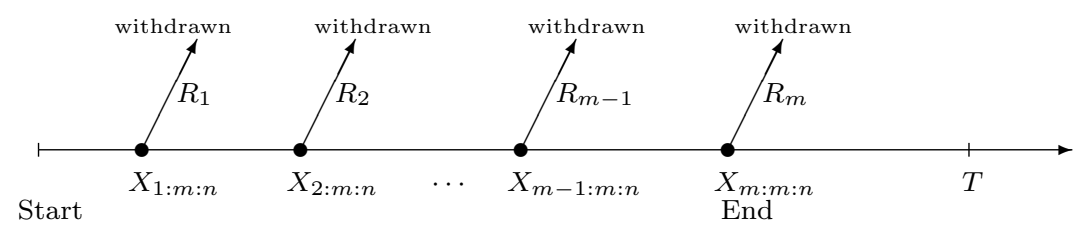

(a) Experiment terminates before time $T$ (i.e. $X_{m: m: n}<T$ )

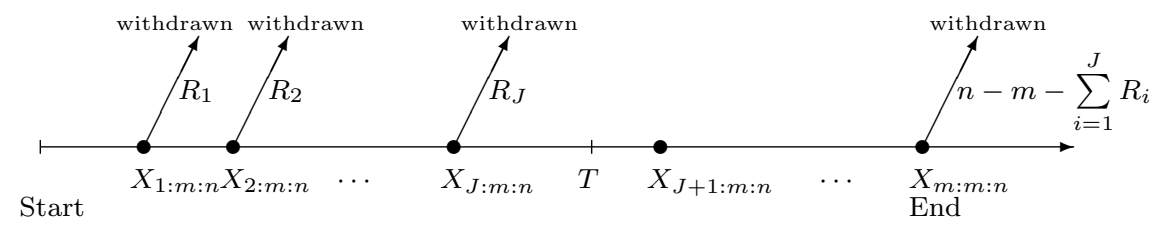

(b) Experiment terminates after time $T$ (i.e. $X_{m: m: n} \geq T$ )

Figure 1: Schematic representation of adaptive Type-II progressive censoring

where $X_{0: m: n} \equiv 0$ and $X_{m+1: m: n} \equiv \infty$. According to the above result on stochastic ordering of first order statistics from different sample sizes, after the experiment passed time $T$, we set $R_{J+1}=\cdots=R_{m-1}=0$ and $R_{m}=n-m-\sum_{i=1}^{J} R_{i}$. This formulation leads us to terminate the experiment as soon as possible if the $(J+1)$-th failure time is greater than $T$ for $J+1<m$. Figure 1(b) gives the schematic representation of this situation. The value of $T$ plays an important role in the determination of the values of $R_{i}$ and also as a compromise between a shorter experimental time and a higher chance to observe extreme failures. One extreme case is when $T \rightarrow \infty$, which means time is not the main consideration for the experimenter, then we will have a usual progressive Type-II censoring scheme with the pre-fixed progressive censoring scheme $\left(R_{1}, R_{2}, \ldots, R_{m}\right)$. Another extreme case can occur when $T=0$, which means we always want to end the experiment as soon as possible, then we will have $R_{1}=\cdots=R_{m-1}=0$ and $R_{m}=n-m$ which results in the conventional Type-II censoring scheme. 


\section{Statistical Inference for Exponential Distribution}

\subsection{Point Estimation}

Given $J=j$, the likelihood function is given by

$$
L(\boldsymbol{\theta} \mid J=j)=d_{j}\left[\prod_{i=1}^{m} f\left(x_{i: m: n} ; \boldsymbol{\theta}\right)\right]\left\{\prod_{i=1}^{j}\left[1-F\left(x_{i: m: n} ; \boldsymbol{\theta}\right)\right]^{R_{i}}\right\}\left[1-F\left(x_{m: m: n} ; \boldsymbol{\theta}\right)\right]\left(n-m-\sum_{i=1}^{j} R_{i}\right)
$$

where

$$
d_{j}=\prod_{i=1}^{m}\left[n-i+1-\sum_{k=1}^{\max \{i-1, j\}} R_{k}\right]
$$

The exponential distribution is one of the most widely used lifetime models in the areas of life testing and reliability. The volume by Balakrishnan and Basu [3] (see also Chapter 19 of [13]) provides an extensive review of the genesis of the distribution and its properties, including several characterization results. For the exponential distribution with $\operatorname{PDF} f(x)=\lambda e^{-\lambda x}, x>0$ and $\operatorname{CDF} F(x)=1-e^{-\lambda x}, x>0($ denoted as $\operatorname{Exp}(\lambda))$, the log-likelihood function is

$$
\begin{aligned}
\ln L(\lambda \mid J=j)= & \text { constant }+m \ln \lambda \\
& -\lambda\left[\sum_{i=1}^{m} x_{i: m: n}+\sum_{i=1}^{j} R_{i} x_{i: m: n}+\left(n-m-\sum_{i=1}^{j} R_{i}\right) x_{m: m: n}\right]
\end{aligned}
$$

and it is maximized at

$$
\hat{\lambda}=\frac{m}{\sum_{i=1}^{m} x_{i: m: n}+\sum_{i=1}^{J} R_{i} x_{i: m: n}+\left(n-m-\sum_{i=1}^{J} R_{i}\right) x_{m: m: n}} .
$$

The maximum likelihood estimator of $\lambda$ is $\hat{\lambda}$ and an estimate of the asymptotic variance is $\widehat{\operatorname{Var}}(\hat{\lambda})=\hat{\lambda}^{2} / m$. For brevity we denote

$$
\delta=\delta(\mathbf{x}, j)=\sum_{i=1}^{m} x_{i: m: n}+\sum_{i=1}^{j} R_{i} x_{i: m: n}+\left(n-m-\sum_{i=1}^{j} R_{i}\right) x_{m: m: n} .
$$

Note that $\delta$ corresponds to the total time on test (TTT) and the MLE of $\lambda$ corresponds to the situation of standard progressive Type-II censoring with censoring scheme $\left(R_{1}, R_{2}, \ldots, R_{J}, 0, \ldots, 0, n-m-\sum_{i=1}^{J} R_{i}\right)$. 


\subsection{Construction of Confidence Interval for $\lambda$}

In this section, we describe six different techniques for constructing $100(1-\alpha) \%$ confidence intervals for the failure rate $\lambda$.

1. Conditional exact confidence interval (EX)

Let $Y$ be a $\operatorname{Gamma}(\alpha, \beta)$, i.e., a gamma random variable with shape parameter $\alpha$ and scale parameter $1 / \beta$. Then the $\mathrm{PDF}$ of $Y$ is given by

$$
g(y ; \alpha, \beta)=\frac{\beta^{\alpha}}{\Gamma(\alpha)} y^{\alpha-1} e^{-\beta y}, \quad y>0
$$

where $\Gamma(\alpha)=\int_{0}^{\infty} x^{\alpha-1} e^{-x} d x$ is the gamma function. Conditional on $J=j$, the exact conditional distribution of $\hat{\lambda}$ is given by (see Appendix $\mathrm{A}$ )

$$
f_{\hat{\lambda}}(u \mid J=j)=\frac{\frac{1}{u^{2}} \sum_{i=1}^{j} \frac{a_{i, j}}{\left(\gamma_{i}-\gamma_{j+1}\right)}\left[e^{-\gamma_{j+1} T \lambda} g\left(\frac{1}{u}-\frac{\gamma_{j+1} T}{m} ; m, m \lambda\right)-e^{-\gamma_{i} T \lambda} g\left(\frac{1}{u}-\frac{\gamma_{i} T}{m} ; m, m \lambda\right)\right]}{\sum_{i=1}^{j} \frac{a_{i, j}}{\left(\gamma_{i}-\gamma_{j+1}\right)}\left[e^{-\gamma_{j+1} T \lambda}-e^{-\gamma_{i} T \lambda}\right]},
$$

$j=0,1,2, \ldots, m$, where

$$
\begin{aligned}
\gamma_{j} & =n-j+1+\sum_{i=1}^{j-1} R_{i}, j=1,2, \ldots, m, \text { with } \gamma_{m+1} \equiv 0, \\
a_{i, j} & =\prod_{\substack{k=1 \\
k \neq i}}^{j} \frac{1}{\gamma_{k}-\gamma_{i}}, 1 \leq i \leq j \leq m,
\end{aligned}
$$

and we use the usual conventions that $\sum_{l=i}^{i-1} d_{l} \equiv 0$.

To construct exact confidence intervals of $\lambda$, we need the assumption, similar to [5], that the probability $\operatorname{Pr}(\hat{\lambda} \geq w \mid J=j)$ is an increasing function of $\lambda$. This assumption guarantees the invertibility of the pivotal quantities and allow us to construct exact confidence intervals of $\lambda$ based on the exact distribution of $\hat{\lambda}$. Several articles including $[5,8,9,12,15]$, have used this approach for constructing exact confidence intervals in different contexts. Plots of $\operatorname{Pr}(\hat{\lambda} \geq w \mid J=j)$ versus $\lambda$ can be used to justify the exact method for specified values of $n, m$ and censoring scheme. In general, the stochastic monotonicity of the MLE for adaptive progressive censoring is a conjecture and it seems 
to be an interesting open problem. Studies on the stochastic monotonicity of MLE based on other censoring schemes can be found in Balakrishnan and Iliopoulos [4].

For $J=j$, let $\lambda_{q}$ be the unique solution of the following nonlinear equation:

$$
\begin{aligned}
& e^{-\gamma_{j+1} T \lambda_{q}}\left[\Gamma\left(m, m \lambda_{q} \max \left(0, \frac{1}{\hat{\lambda}}-\frac{T \gamma_{j+1}}{m}\right)\right)-(1-q)\right]\left[\sum_{i=1}^{j} \frac{a_{i, j}}{\left(\gamma_{i}-\gamma_{j+1}\right)}\right] \\
& -\sum_{i=1}^{j} \frac{a_{i, j}}{\left(\gamma_{i}-\gamma_{j+1}\right)} e^{-\gamma_{i} T \lambda_{q}}\left[\Gamma\left(m, m \lambda_{q} \max \left(0, \frac{1}{\hat{\lambda}}-\frac{T \gamma_{i}}{m}\right)\right)-(1-q)\right]=0
\end{aligned}
$$

where $\Gamma(m, z)=\frac{1}{\Gamma(m)} \int_{z}^{\infty} t^{m-1} \exp (-t) d t$. Then, the conditional $100(1-\alpha) \%$ confidence interval for $\lambda$ can be obtained as $\left(\lambda_{\alpha / 2}, \lambda_{1-\alpha / 2}\right)$.

2. Normal approximation of the MLE (NA)

Since the standard regularity conditions for the asymptotic properties of MLE are satisfied by the exponential distribution (see, for example, Meeker and Escobar [17]), for large value of effective sample size $m$, we have

$$
\frac{\hat{\lambda}-\lambda}{\sqrt{\operatorname{Var}(\hat{\lambda})}} \dot{\sim} N(0,1) .
$$

If we replace the variance $\operatorname{Var}(\hat{\lambda})$ by its estimate, we can obtain an approximate $100(1-$ $\alpha) \%$ confidence interval for $\lambda$ as

$$
\hat{\lambda} \pm z_{1-\alpha / 2} \frac{\hat{\lambda}}{\sqrt{m}}
$$

where $z_{q}$ is the $100 q$-th percentile of a standard normal distribution.

3. Normal approximation of the log-transformed MLE (NL)

The problem with applying normal approximation of the MLE is that when the sample size is small, the normal approximation may be poor. However, a different transformation of the MLE can be used to correct the inadequate performance of the normal approximation. Since the parameter of interest, $\lambda$, is a positive parameter, log-transformation can be considered. Based on the normal approximation of the log-transformed MLE (Meeker and Escobar [17]) and $\operatorname{Var}(\ln \hat{\lambda})$ can be approximation by delta method as $\widehat{\operatorname{Var}}(\ln \hat{\lambda})=1 / m$, 
an approximate $100(1-\alpha) \%$ confidence interval for $\lambda$ is

$$
\left[\frac{\hat{\lambda}}{\exp \left(z_{1-\alpha / 2} \sqrt{\frac{1}{m}}\right)}, \hat{\lambda} \cdot \exp \left(z_{1-\alpha / 2} \sqrt{\frac{1}{m}}\right)\right] .
$$

4. Likelihood ratio-based confidence interval (LR)

A likelihood ratio-based conditional confidence interval is constructed using the likelihood ratio statistic [18] for testing the hypothesis $H_{0}: \lambda=\lambda_{0}$ versus $H_{1}: \lambda \neq \lambda_{0}$. In our case, the likelihood ratio statistic is given by $2\left[\ln L(\hat{\lambda} \mid J=j)-\ln L\left(\lambda_{0} \mid J=j\right)\right]$.

The asymptotic distribution of the likelihood ratio statistic is chi-square with one degree of freedom $\left(\chi_{1}^{2}\right)$. An approximate $100(1-\alpha) \%$ confidence interval for $\lambda$ is the region

$$
\left\{\lambda: 2[\ln L(\hat{\lambda} \mid J=j)-\ln L(\lambda \mid J=j)] \leq \chi_{1,1-\alpha}^{2}\right\},
$$

where $\chi_{\nu, q}^{2}$ is the $100 q$-th percentile of a chi-square distribution with $\nu$ degrees of freedom.

5. Bootstrap confidence interval (PB and $\mathrm{TB})$

We construct confidence intervals based on the parametric bootstrap using the percentile bootstrap method and bootstrap $t$ method (see, for example, [11]). To obtain the percentile bootstrap confidence intervals for $\lambda$, we use the following algorithm:

Parametric percentile bootstrap confidence interval (PB):

1. Based on the original sample $\mathbf{x}=\left(x_{1: m: n}, x_{2: m: n}, \ldots, x_{m: m: n}\right)$, obtain $\hat{\lambda}$, the MLE of $\lambda$.

2. Simulate the adaptive Type-II progressively censored sample, say $\left(y_{1: m: n}, \ldots, y_{m: m: n}\right)$, with the underlying distribution as $\operatorname{Exp}(\hat{\lambda})$ (simulation algorithm is described in the following section) with censoring scheme $\left(R_{1}, R_{2}, \ldots, R_{m}\right)$ and pre-fixed $T$.

3. Compute the MLE of $\lambda$ based on $y_{1: m: n}, y_{2: m: n}, \ldots, y_{m: m: n}$, say $\hat{\lambda}^{*}$.

4. Repeat Steps $2-3 B$ times and obtain $\hat{\lambda}^{*(1)}, \hat{\lambda}^{*(2)}, \ldots, \hat{\lambda}^{*(B)}$.

5. Arrange $\hat{\lambda}^{*(1)}, \hat{\lambda}^{*(2)}, \ldots, \hat{\lambda}^{*(B)}$ in ascending order and obtain $\hat{\lambda}^{*[1]}, \hat{\lambda}^{*[2]}, \ldots, \hat{\lambda}^{*[B]}$.

A two-sided $100(1-\alpha) \%$ percentile bootstrap confidence interval of $\lambda$, say $\left[\lambda_{L}^{*}, \lambda_{U}^{*}\right]$, is then given by $\left(\lambda_{L}^{*}, \lambda_{U}^{*}\right)=\left(\hat{\lambda}^{*([B \alpha / 2])}, \hat{\lambda}^{*([B(1-\alpha / 2)])}\right)$. 
To obtain the bootstrap- $t$ confidence intervals for $\lambda$, we use the following algorithm:

Parametric bootstrap- $t$ confidence interval (TB):

$1-3$. Same as the steps $1-3$ above.

4. Compute the $t$-statistic $T=\left[\sqrt{m}\left(\hat{\lambda}^{*}-\hat{\lambda}\right)\right] / \hat{\lambda}^{*}$.

5. Repeat Steps $2-4 B$ times and obtain $T^{(1)}, T^{(2)}, \ldots, T^{(B)}$.

6. Arrange $T^{(1)}, T^{(2)}, \ldots, T^{(B)}$ in ascending order and obtain $T^{[1]}, T^{[2]}, \ldots, T^{[B]}$.

A two-sided $100(1-\alpha) \%$ bootstrap- $t$ confidence interval of $\lambda$, say $\left[\lambda_{t, L}, \lambda_{t, U}^{*}\right]$, is then given by $\left(\lambda_{t, L}, \lambda_{t, U}\right)=\left(\hat{\lambda}+T^{([B \alpha / 2])} \frac{\hat{\lambda}}{\sqrt{m}}, \hat{\lambda}+T^{([B(1-\alpha / 2)])} \frac{\hat{\lambda}}{\sqrt{m}}\right)$.

6. Bayesian Analysis (BN and BA)

Bayesian inference provides an alternative way to estimate the parameter $\lambda$, especially when prior information about $\lambda$ is available. A flexible choice of prior distribution for $\lambda$ is a gamma prior with parameters $a$ and $b$ (denote by $\operatorname{Gamma}(a, b)$ ). Given the data, the posterior density of $\lambda$ is proportional to $\lambda^{a+m-1} \exp (-\lambda \delta)$, which is the kernel of Gamma $(a+m, b+\delta)$. Therefore, if we assume the commonly used squared error loss function, the Bayes estimator of $\lambda$ is given by $\tilde{\lambda}=(a+m) /(b+\delta)$. A $100(1-\alpha) \%$ Bayesian credible interval, say $\left(\tilde{\lambda}_{\alpha / 2}, \tilde{\lambda}_{1-\alpha / 2}\right)$, can be obtained as the solution of the following equation:

$$
\int_{0}^{\tilde{\lambda}_{q}} \frac{(b+\delta)^{(a+m)}}{\Gamma(a+m)} \lambda^{a+m-1} \exp (-(b+\delta) \lambda) d \lambda=q .
$$

If $a$ is an integer, a $100(1-\alpha) \%$ Bayesian credible interval can be obtained as

$$
\left(\frac{\chi_{2(a+m), \alpha / 2}^{2}}{2(b+\delta)}, \frac{\chi_{2(a+m), 1-\alpha / 2}^{2}}{2(b+\delta)}\right) \text {. }
$$

In the simulation study presented in Section 4 below, we use the non-informative prior with $a=b=0$ (say, BN) and prior distribution $\operatorname{Gamma}(0.1,0.1)$ (say, BA).

\subsection{Expected Total Test Time}

As we mentioned before, the difference between the proposed adaptive Type-II progressive censoring scheme and the one proposed by Kundu and Joarder [16] is that the maximum total 
test time is not fixed in advance. In practical applications, it is useful to have the average total test time for a particular life testing plan. When an adaptive Type-II progressive censoring scheme is used, one can obtain the expected total test time (ETT) by

$$
E T T=E\left(X_{m: m: n}\right)=\sum_{j=1}^{m} \operatorname{Pr}(J=j) E\left(X_{m: m: n} \mid J=j\right) .
$$

For exponential distribution, we can show that the probability mass function of the value $J$ for a pre-fixed value of $T$ is (see Appendix B)

$$
\begin{aligned}
\operatorname{Pr}(J=j) & =\operatorname{Pr}\left(X_{j: m: n}<T \leq X_{j+1: m: n}\right) \\
& =c_{j-1} \exp \left(-\gamma_{j+1} \lambda T\right) \sum_{i=1}^{j} \frac{a_{i, j}}{\left(\gamma_{i}-\gamma_{j+1}\right)}\left\{1-\exp \left[-\left(\gamma_{i}-\gamma_{j+1}\right) \lambda T\right]\right\},
\end{aligned}
$$

$j=0,1,2, \ldots, m$, where $\gamma_{j}$ and $a_{i, j}$ are given in equations (2) and (3), respectively, and

$$
c_{j-1}=\prod_{i=1}^{j} \gamma_{i}, j=1,2, \ldots, m, \text { with } c_{0} \equiv 1
$$

Based on the memoryless property of exponential distribution and the properties of exponential order statistics, we can obtain the conditional expectation of $X_{m: m: n}$ for $j=0,1, \ldots, m-1$ as

$$
E\left(X_{m: m: n} \mid J=j\right)=T+E\left(Y_{r: s}\right)=T+\frac{1}{\lambda} \sum_{k=s-r+1}^{s} \frac{1}{k}
$$

where $Y_{r: s}$ is the $r$-th order statistic from independently and identically distributed exponential random variables with mean $1 / \lambda$ and sample size $s, s=n-j-\sum_{i=1}^{j} R_{i}$ and $r=m-j$ (see, for example, David and Nagaraja [10]). For $j=m$, the conditional expectation of $X_{m: m: n}$ is

$$
\begin{aligned}
E\left(X_{m: m: n} \mid J=m\right) & =\int_{0}^{T} \frac{x f_{X_{m: m: n}}(x)}{F_{X_{m: m: n}}(T)} d x \\
& =\frac{c_{m-1} \sum_{i=1}^{m} \frac{a_{i, m}}{\gamma_{i}}\left[\frac{1-\exp \left(-\gamma_{i} \lambda T\right)}{\gamma_{i} \lambda}-T \exp \left(-\gamma_{i} \lambda T\right)\right]}{\operatorname{Pr}(J=m)} .
\end{aligned}
$$

Some information on $\lambda$ from past data or prior experience is typically available. Therefore, from equations (5), (7) and (8), we can approximate the expected test length provided that the values of $n$ and $m$, the progressive censoring scheme $\left(R_{1}, R_{2}, \ldots, R_{m}\right)$ and $T$ are specified. 


\section{Monte Carlo Simulation and Numerical Comparisons}

We first describe the procedure to generate Type-II progressively censored data (from any distribution $F$ ) for given values of $n, m, T$ and $\left(R_{1}, R_{2}, \ldots, R_{m}\right)$ :

1. Generate an ordinary Type-II progressively censored sample $X_{1: m: n}, X_{2: m: n}, \ldots, X_{m: m: n}$ with censoring scheme $\left(R_{1}, R_{2}, \ldots, R_{m}\right)$ based on the method proposed in [6].

2. Determine the value of $J$, where $X_{J: m: n}<T<X_{J+1: m: n}$, and discard the sample $X_{j+2: m: n}, \ldots, X_{m: m: n}$.

3. Generate the first $m-j-1$ order statistics from a truncated distribution $f(x) /[1-$ $\left.F\left(x_{j+1: m: n}\right)\right]$ with sample size $\left(n-\sum_{i=1}^{j} R_{i}-j-1\right)$ as $X_{j+2: m: n}, X_{j+3: m: n}, \ldots, X_{m: m: n}$.

For the exponential distribution, a more convenient algorithm based on spacings of progressively censored exponential order statistics (Balakrishnan and Aggarwala [2], Section 3.3) can be used in place of Step 1 above.

\subsection{Comparison of two progressive censoring schemes}

In this subsection, we compare the efficiency of the MLE based on the adaptive Type-II progressive censoring scheme with the hybrid censoring scheme proposed by Kundu and Joarder [16]. If the censoring scheme proposed in [16] is employed, the MLE is given by

$$
\hat{\lambda}_{K J}= \begin{cases}\frac{J}{\sum_{i=1}^{J}\left(R_{i}+1\right) x_{i: m: n}+T\left(n-J-\sum_{i=1}^{J} R_{i}\right)} & x_{m: m: n}>T, \\ \sum_{i=1}^{m}\left(R_{i}+1\right) x_{i: m: n} & x_{m: m: n} \leq T .\end{cases}
$$

The expected total test time and expected number of failures based on the censoring scheme proposed in [16] can be computed respectively by

$$
\begin{aligned}
E T T_{K J} & =\operatorname{Pr}(J=m) E\left(X_{m: m: n} \mid J=m\right)+[1-\operatorname{Pr}(J=m)] T \\
\text { and } E M_{K J} & =\sum_{j=1}^{m} j \operatorname{Pr}(J=j) .
\end{aligned}
$$

Monte Carlo simulation is used to compare the efficiency of the MLE from the two censoring schemes. Different values of $n, m$ and $T$ and three progressive censoring schemes for each setting 
are considered. For brevity, for example, the censoring scheme $(0,0,1,1,1,1,1,0,0,0)$ is denoted by $\left(0^{*} 2,1^{*} 5,0^{*} 3\right)$. Without loss of generality, we set $\lambda=1$. The biases and mean squared errors (MSEs) for $m=5$ are estimated based on 10,000 simulations and they are reported in Table 1 . We presented only the representative results for $m=5$ here due to space limitations, but the interested reader may obtain the simulation results for other sample sizes from the authors. For the sake of comparison, based on exact calculation, the expected total test time for both censoring schemes, the expected effective sample size for scheme proposed in [16] and the probability of getting no observations (i.e. $\operatorname{Pr}(J=0))$ are also presented in Table 1 .

From Table 1, we observed that the MLEs based on the adaptive Type-II progressive censoring schemes give larger biases but smaller MSEs compared to those based on the hybrid censoring scheme proposed in [16]. Although the proposed censoring scheme gives better performance in estimation in terms of MSE, the trade-off is a longer experimental time and a larger effective sample size. Therefore, the proposed censoring scheme will be useful to obtain a higher efficiency in parameter estimation when the length of the experiment is not a major concern.

In studying the effect of progressive censoring schemes on the efficiency of estimation, we observed that the MSEs are close for the three chosen censoring schemes for each set of $n$ and $m$, however, the expected total test times can be very different for different progressive censoring schemes. For example, in Table $1,(n, m)=(50,5)$ with $T=0.25$, the MSEs for censoring schemes $(0,0,0,0,45),(45,0,0,0,0)$ and $(9,9,9,9,9)$ are $0.5931,0.5705$ and 0.5861 and their corresponding expected total test times are 0.1043, 2.1033 and 0.2255 , respectively. This suggests that for a significant reduction in the testing time without sacrificing much in efficiency of estimation, one should use the conventional Type-II censoring scheme and avoid the use of censoring schemes with heavy censoring at the early stages of the experiment.

\subsection{Comparison of methods for confidence interval construction}

In this subsection, Monte Carlo simulation was employed to investigate the performance of different confidence interval construction methods. Criteria appropriate to the evaluation of 
Table 1: Biases and MSEs of the MLEs for different sample sizes and censoring schemes for $m=5$

\begin{tabular}{|c|c|c|c|c|c|c|c|c|c|}
\hline$(n, m)$ & Scheme & Bias & $M S E$ & $E T T$ & Bias $_{K J}$ & $M S E_{K J}$ & $E T T_{K J}$ & $E M_{K J}$ & $\operatorname{Pr}(J=0)$ \\
\hline \multirow[t]{12}{*}{$(15,5)$} & & \multicolumn{8}{|c|}{$T=0.25$} \\
\hline & $(0,0,0,0,10)$ & 0.2536 & 0.6210 & 0.3916 & 0.1507 & 0.6765 & 0.2360 & 3.1848 & 0.0235 \\
\hline & $(10,0,0,0,0)$ & 0.2336 & 0.5365 & 2.1109 & 0.2855 & 1.0113 & 0.2499 & 1.6455 & 0.0235 \\
\hline & $(2,2,2,2,2)$ & 0.2501 & 0.5690 & 0.6023 & 0.1882 & 0.6634 & 0.2478 & 2.6382 & 0.0235 \\
\hline & & \multicolumn{8}{|c|}{$T=0.50$} \\
\hline & $(0,0,0,0,10)$ & 0.2455 & 0.6442 & 0.3893 & 0.2217 & 0.6664 & 0.3570 & 4.6313 & 0.0006 \\
\hline & $(10,0,0,0,0)$ & 0.2477 & 0.5357 & 2.1491 & 0.1934 & 0.6936 & 0.4983 & 2.4002 & 0.0006 \\
\hline & $(2,2,2,2,2)$ & 0.2619 & 0.6070 & 0.7113 & 0.2084 & 0.6282 & 0.4609 & 3.8843 & 0.0006 \\
\hline & & \multicolumn{8}{|c|}{$T=1.00$} \\
\hline & $(0,0,0,0,10)$ & 0.2513 & 0.5788 & 0.3893 & 0.2509 & 0.5793 & 0.3888 & 4.9946 & 0.0000 \\
\hline & $(10,0,0,0,0)$ & 0.2615 & 0.6118 & 2.1500 & 0.2007 & 0.6479 & 0.9651 & 3.4234 & 0.0000 \\
\hline & $(2,2,2,2,2)$ & 0.2451 & 0.5573 & 0.7580 & 0.2304 & 0.5669 & 0.6821 & 4.7511 & 0.0000 \\
\hline \multirow[t]{12}{*}{$(25,5)$} & & \multicolumn{8}{|c|}{$T=0.25$} \\
\hline & $(0,0,0,0,20)$ & 0.2480 & 0.5924 & 0.2183 & 0.2094 & 0.6264 & 0.1919 & 4.4330 & 0.0019 \\
\hline & $(20,0,0,0,0)$ & 0.2389 & 0.5366 & 2.1198 & 0.2561 & 0.9535 & 0.2499 & 1.7534 & 0.0019 \\
\hline & $(4,4,4,4,4)$ & 0.2436 & 0.5457 & 0.4068 & 0.1837 & 0.5803 & 0.2382 & 3.5675 & 0.0019 \\
\hline & & \multicolumn{8}{|c|}{$T=0.50$} \\
\hline & $(0,0,0,0,20)$ & 0.2526 & 0.5577 & 0.2182 & 0.2518 & 0.5588 & 0.2175 & 4.9853 & 0.0000 \\
\hline & $(20,0,0,0,0)$ & 0.2448 & 0.6059 & 2.1233 & 0.1911 & 0.7457 & 0.4979 & 2.4728 & 0.0000 \\
\hline & $(4,4,4,4,4)$ & 0.2472 & 0.6198 & 0.4513 & 0.2231 & 0.6328 & 0.3810 & 4.5896 & 0.0000 \\
\hline & & \multicolumn{8}{|c|}{$T=1.00$} \\
\hline & $(0,0,0,0,20)$ & 0.2496 & 0.5793 & 0.2182 & 0.2496 & 0.5793 & 0.2182 & 5.0000 & 0.0000 \\
\hline & $(20,0,0,0,0)$ & 0.2584 & 0.5938 & 2.1233 & 0.2009 & 0.6297 & 0.9618 & 3.4672 & 0.0000 \\
\hline & $(4,4,4,4,4)$ & 0.2544 & 0.5877 & 0.4566 & 0.2524 & 0.5897 & 0.4500 & 4.9663 & 0.0000 \\
\hline \multirow[t]{12}{*}{$(50,5)$} & & \multicolumn{8}{|c|}{$T=0.25$} \\
\hline & $(0,0,0,0,45)$ & 0.2469 & 0.5931 & 0.1043 & 0.2463 & 0.5939 & 0.1040 & 4.9897 & 0.0000 \\
\hline & $(45,0,0,0,0)$ & 0.2593 & 0.5705 & 2.1033 & 0.2964 & 1.0349 & 0.2499 & 1.8212 & 0.0000 \\
\hline & $(9,9,9,9,9)$ & 0.2545 & 0.5861 & 0.2255 & 0.2318 & 0.5984 & 0.1905 & 4.5896 & 0.0000 \\
\hline & & \multicolumn{8}{|c|}{$T=0.50$} \\
\hline & $(0,0,0,0,45)$ & 0.2497 & 0.5724 & 0.1043 & 0.2497 & 0.5724 & 0.1043 & 5.0000 & 0.0000 \\
\hline & $(45,0,0,0,0)$ & 0.2511 & 0.5645 & 2.1033 & 0.1999 & 0.7010 & 0.4976 & 2.5244 & 0.0000 \\
\hline & $(9,9,9,9,9)$ & 0.2440 & 0.5437 & 0.2283 & 0.2417 & 0.5459 & 0.2250 & 4.9663 & 0.0000 \\
\hline & & \multicolumn{8}{|c|}{$T=1.00$} \\
\hline & $(0,0,0,0,45)$ & 0.2464 & 0.5307 & 0.1043 & 0.2464 & 0.5307 & 0.1043 & 5.0000 & 0.0000 \\
\hline & $(45,0,0,0,0)$ & 0.2419 & 0.5590 & 2.1033 & 0.1820 & 0.5919 & 0.9591 & 3.4985 & 0.0000 \\
\hline & $(9,9,9,9,9)$ & 0.2560 & 0.6227 & 0.2283 & 0.2560 & 0.6227 & 0.2283 & 4.9998 & 0.0000 \\
\hline
\end{tabular}


the various methods under scrutiny include: closeness of the coverage probability to its nominal value and expected interval width. For each simulated sample under a particular setting, we computed $95 \%$ confidence intervals and checked whether the true value lay within the interval and recorded the length of the confidence interval. This procedure was repeated 10,000 times. The estimated coverage probability was computed as the number of confidence intervals that covered the true values divided by 10,000 while the estimated expected width of the confidence interval was computed as the sum of the lengths for all intervals divided by 10,000. The coverage probabilities and the expected widths for different sample sizes, censoring schemes and $T=0.25,0.5$ and 1.0 are presented in Tables 2 and 3 . We presented only the representative results here due to space limitations, but the interested reader may obtain the simulation results for other sample sizes from the authors.

When comparing in terms of coverage probabilities, EX, NA, LR, PB, BN and BA maintain the coverage probabilities close to or above the nominal level in all the situations considered here. We observed that the exact confidence interval (EX) has coverage probabilities always above the nominal level, however, its expected width is the largest among all the interval estimation procedures considered here. Among these methods, BA has the shortest expected widths followed by BN. On the other hand, we observed that the TB method produces the shortest expected width among all the methods but its coverage probabilities may not be maintained at the nominal level in some situations.

For the Bayesian credible interval, we tried different prior distributions with different values of $a$ and $b$ and found that a prior distribution with correct information (for example, $a=1$, $b=1$ have $E(\lambda)=1$ is the true value) about the true value of $\lambda$ improves the performance of the Bayesian credible interval compared to the one using non-informative prior $(a=b=0)(\mathrm{BN})$, however, a prior distribution not matched with the true value of $\lambda$ (for example, $a=2, b=4$ ) degrades the performance of the Bayesian credible interval. We presented here the results for prior distributions with $a=b=0$ and $a=b=0.1$ for illustrative purposes.

In terms of computational effort, the normal approximation confidence interval (NA) and the Bayesian credible intervals (BN and BA) can be easily computed with hand calculator and statistical tables while computer programs are required for the other confidence intervals. 
Table 2: Coverage probabilities and expected width of $95 \%$ confidence intervals based on different methods for $(n, m)=(15,5)$ and $(25,5)$

\begin{tabular}{|c|c|c|c|c|c|c|c|c|}
\hline \multirow[b]{2}{*}{$(n, m)$} & \multirow[b]{2}{*}{ Scheme } & \multirow[b]{2}{*}{ Method } & \multicolumn{2}{|c|}{$T=0.25$} & \multicolumn{2}{|c|}{$T=0.5$} & \multicolumn{2}{|c|}{$T=1.00$} \\
\hline & & & Coverage & Length & Coverage & Length & Coverage & Length \\
\hline \multirow[t]{24}{*}{$(15,5)$} & $(0,0,0,0,10)$ & $\mathrm{EX}$ & 97.70 & 5.097 & 95.55 & 3.009 & 95.03 & 2.093 \\
\hline & & NA & 95.72 & 2.147 & 95.67 & 2.136 & 95.44 & 2.153 \\
\hline & & NL & 93.25 & 2.092 & 93.31 & 2.087 & 93.11 & 2.081 \\
\hline & & LR & 94.83 & 1.998 & 94.62 & 1.982 & 94.59 & 1.984 \\
\hline & & $\mathrm{PB}$ & 99.54 & 2.549 & 95.00 & 2.388 & 94.90 & 2.399 \\
\hline & & TВ & 88.65 & 1.262 & 88.30 & 1.218 & 87.47 & 1.193 \\
\hline & & $\mathrm{BN}$ & 95.22 & 1.970 & 95.01 & 1.956 & 94.96 & 1.961 \\
\hline & & $\mathrm{BA}$ & 95.30 & 1.966 & 95.05 & 1.951 & 95.00 & 1.956 \\
\hline & $(10,0,0,0,0)$ & EX & 95.41 & 2.509 & 96.48 & 2.891 & 95.94 & 3.004 \\
\hline & & NA & 95.69 & 2.123 & 95.94 & 2.152 & 95.66 & 2.167 \\
\hline & & NL & 93.75 & 2.086 & 93.35 & 2.093 & 92.98 & 2.088 \\
\hline & & LR & 94.94 & 1.972 & 94.82 & 1.993 & 94.41 & 1.988 \\
\hline & & PB & 100.00 & 2.684 & 100.00 & 2.677 & 99.49 & 2.599 \\
\hline & & ТВ & 93.85 & 1.466 & 92.44 & 1.384 & 89.33 & 1.273 \\
\hline & & $\mathrm{BN}$ & 95.17 & 1.944 & 95.12 & 1.969 & 94.97 & 1.971 \\
\hline & & $\mathrm{BA}$ & 95.20 & 1.938 & 95.15 & 1.963 & 95.05 & 1.967 \\
\hline & $(2,2,2,2,2)$ & $\mathrm{EX}$ & 95.20 & 3.355 & 97.31 & 2.979 & 95.86 & 2.357 \\
\hline & & NA & 95.91 & 2.154 & 95.50 & 2.163 & 95.67 & 2.142 \\
\hline & & NL & 93.37 & 2.094 & 92.86 & 2.091 & 93.12 & 2.080 \\
\hline & & LR & 94.58 & 1.979 & 94.26 & 1.991 & 94.42 & 1.977 \\
\hline & & $\mathrm{PB}$ & 99.98 & 2.646 & 98.20 & 2.510 & 94.85 & 2.406 \\
\hline & & ТВ & 91.38 & 1.338 & 88.00 & 1.243 & 87.61 & 1.198 \\
\hline & & $\mathrm{BN}$ & 95.05 & 1.954 & 94.74 & 1.972 & 94.75 & 1.948 \\
\hline & & $\mathrm{BA}$ & 95.08 & 1.949 & 94.81 & 1.968 & 94.78 & 1.943 \\
\hline \multirow[t]{24}{*}{$(25,5)$} & $(0,0,0,0,20)$ & $\mathrm{EX}$ & 96.91 & 3.486 & 95.70 & 2.101 & 95.52 & 2.039 \\
\hline & & NA & 95.37 & 2.139 & 95.60 & 2.159 & 95.56 & 2.146 \\
\hline & & NL & 93.16 & 2.075 & 92.78 & 2.077 & 93.32 & 2.095 \\
\hline & & LR & 94.33 & 1.968 & 94.49 & 1.987 & 94.75 & 1.991 \\
\hline & & $\mathrm{PB}$ & 94.61 & 2.387 & 94.91 & 2.395 & 95.02 & 2.423 \\
\hline & & $\mathrm{TB}$ & 87.65 & 1.215 & 87.09 & 1.189 & 87.53 & 1.187 \\
\hline & & $\mathrm{BN}$ & 94.67 & 1.945 & 94.99 & 1.967 & 95.00 & 1.961 \\
\hline & & $\mathrm{BA}$ & 94.70 & 1.940 & 95.08 & 1.964 & 95.05 & 1.955 \\
\hline & $(20,0,0,0,0)$ & $\mathrm{EX}$ & 95.28 & 2.450 & 96.24 & 2.759 & 95.40 & 2.931 \\
\hline & & NA & 95.46 & 2.130 & 95.33 & 2.131 & 95.86 & 2.160 \\
\hline & & NL & 93.39 & 2.081 & 93.23 & 2.066 & 93.40 & 2.100 \\
\hline & & LR & 94.69 & 1.978 & 94.41 & 1.966 & 94.74 & 1.995 \\
\hline & & $\mathrm{PB}$ & 100.00 & 2.689 & 99.99 & 2.716 & 99.52 & 2.590 \\
\hline & & ТВ & 93.57 & 1.458 & 91.84 & 1.362 & 89.64 & 1.283 \\
\hline & & $\mathrm{BN}$ & 95.11 & 1.957 & 94.64 & 1.933 & 95.14 & 1.972 \\
\hline & & $\mathrm{BA}$ & 95.19 & 1.953 & 94.69 & 1.928 & 95.22 & 1.969 \\
\hline & $(4,4,4,4,4)$ & $\mathrm{EX}$ & 96.78 & 3.344 & 96.02 & 2.629 & 95.11 & 2.147 \\
\hline & & NA & 95.70 & 2.140 & 95.63 & 2.135 & 95.25 & 2.151 \\
\hline & & NL & 93.44 & 2.087 & 93.63 & 2.091 & 93.08 & 2.087 \\
\hline & & LR & 94.42 & 1.971 & 94.74 & 1.981 & 94.34 & 1.987 \\
\hline & & PB & 99.16 & 2.570 & 95.71 & 2.434 & 94.53 & 2.383 \\
\hline & & ТВ & 89.30 & 1.272 & 88.23 & 1.215 & 87.14 & 1.195 \\
\hline & & $\mathrm{BN}$ & 94.94 & 1.949 & 95.09 & 1.951 & 94.56 & 1.958 \\
\hline & & BA & 95.02 & 1.945 & 95.14 & 1.947 & 94.66 & 1.955 \\
\hline
\end{tabular}


Table 3: Coverage probabilities and expected width of $95 \%$ confidence intervals based on different methods for $(n, m)=(15,10)$ and $(25,10)$

\begin{tabular}{|c|c|c|c|c|c|c|c|c|}
\hline \multirow[b]{2}{*}{$(n, m)$} & \multirow[b]{2}{*}{ Scheme } & \multirow[b]{2}{*}{ Method } & \multicolumn{2}{|c|}{$T=0.25$} & \multicolumn{2}{|c|}{$T=0.5$} & \multicolumn{2}{|c|}{$T=1.00$} \\
\hline & & & Coverage & Length & Coverage & Length & Coverage & Length \\
\hline \multirow[t]{24}{*}{$(15,10)$} & $(0 * 9,5)$ & $\mathrm{EX}$ & 95.60 & 2.128 & 96.29 & 2.250 & 96.80 & 2.329 \\
\hline & & NA & 95.52 & 1.328 & 95.85 & 1.335 & 95.87 & 1.330 \\
\hline & & NL & 93.96 & 1.319 & 93.79 & 1.314 & 94.38 & 1.324 \\
\hline & & LR & 94.69 & 1.283 & 94.62 & 1.279 & 95.20 & 1.289 \\
\hline & & PB & 100.00 & 1.548 & 99.91 & 1.522 & 96.15 & 1.413 \\
\hline & & ТВ & 97.42 & 1.191 & 94.85 & 1.102 & 92.08 & 1.021 \\
\hline & & $\mathrm{BN}$ & 94.95 & 1.273 & 94.93 & 1.271 & 95.43 & 1.280 \\
\hline & & BA & 94.98 & 1.271 & 95.01 & 1.271 & 95.49 & 1.279 \\
\hline & $(5,0 * 9)$ & $\mathrm{EX}$ & 95.56 & 1.547 & 96.39 & 1.650 & 96.00 & 1.871 \\
\hline & & NA & 95.53 & 1.332 & 95.56 & 1.326 & 95.27 & 1.323 \\
\hline & & NL & 93.90 & 1.319 & 94.26 & 1.320 & 94.06 & 1.316 \\
\hline & & LR & 94.90 & 1.288 & 95.00 & 1.284 & 94.57 & 1.278 \\
\hline & & $\mathrm{PB}$ & 100.00 & 1.506 & 100.00 & 1.530 & 99.70 & 1.522 \\
\hline & & ТВ & 97.75 & 1.224 & 97.03 & 1.164 & 94.57 & 1.087 \\
\hline & & $\mathrm{BN}$ & 95.19 & 1.280 & 95.25 & 1.274 & 94.82 & 1.269 \\
\hline & & $\mathrm{BA}$ & 95.20 & 1.279 & 95.31 & 1.274 & 94.87 & 1.269 \\
\hline & $(0 * 2,1 * 5,0 * 3)$ & $\mathrm{EX}$ & 96.22 & 1.714 & 96.00 & 1.963 & 96.02 & 1.959 \\
\hline & & NA & 95.46 & 1.328 & 95.48 & 1.328 & 95.54 & 1.325 \\
\hline & & NL & 94.23 & 1.323 & 94.06 & 1.320 & 94.29 & 1.320 \\
\hline & & LR & 95.05 & 1.289 & 94.70 & 1.280 & 94.94 & 1.282 \\
\hline & & PB & 100.00 & 1.514 & 99.98 & 1.519 & 99.07 & 1.502 \\
\hline & & TB & 97.52 & 1.198 & 95.51 & 1.124 & 93.89 & 1.065 \\
\hline & & $\mathrm{BN}$ & 95.34 & 1.280 & 95.06 & 1.273 & 95.07 & 1.272 \\
\hline & & $\mathrm{BA}$ & 95.37 & 1.279 & 95.07 & 1.271 & 95.08 & 1.271 \\
\hline \multirow[t]{24}{*}{$(25,10)$} & $(0 * 9,15)$ & EX & 98.10 & 2.708 & 96.80 & 2.374 & 94.37 & 1.350 \\
\hline & & NA & 95.44 & 1.322 & 95.71 & 1.336 & 95.23 & 1.322 \\
\hline & & NL & 94.03 & 1.313 & 94.02 & 1.324 & 94.08 & 1.319 \\
\hline & & LR & 94.91 & 1.281 & 94.97 & 1.293 & 94.55 & 1.279 \\
\hline & & PB & 99.93 & 1.536 & 95.59 & 1.396 & 94.62 & 1.398 \\
\hline & & $\mathrm{TB}$ & 95.40 & 1.105 & 91.50 & 1.017 & 91.54 & 0.999 \\
\hline & & $\mathrm{BN}$ & 95.03 & 1.270 & 95.25 & 1.283 & 94.82 & 1.270 \\
\hline & & $\mathrm{BA}$ & 95.04 & 1.269 & 95.29 & 1.282 & 94.84 & 1.269 \\
\hline & $(15,0 * 9)$ & EX & 97.98 & 1.589 & 97.00 & 1.737 & 97.10 & 1.905 \\
\hline & & NA & 95.76 & 1.325 & 95.78 & 1.334 & 95.49 & 1.322 \\
\hline & & NL & 94.72 & 1.328 & 93.78 & 1.316 & 94.07 & 1.313 \\
\hline & & LR & 95.31 & 1.288 & 94.67 & 1.282 & 94.78 & 1.276 \\
\hline & & PB & 100.00 & 1.510 & 100.00 & 1.525 & 99.70 & 1.524 \\
\hline & & TB & 97.88 & 1.218 & 96.62 & 1.161 & 94.39 & 1.082 \\
\hline & & $\mathrm{BN}$ & 95.30 & 1.273 & 95.07 & 1.276 & 94.86 & 1.264 \\
\hline & & BA & 95.33 & 1.272 & 95.12 & 1.275 & 94.90 & 1.263 \\
\hline & $(1 * 5,2 * 5)$ & EX & 95.01 & 2.039 & 94.00 & 2.066 & 95.12 & 1.681 \\
\hline & & NA & 94.97 & 1.326 & 95.66 & 1.328 & 95.89 & 1.341 \\
\hline & & NL & 93.94 & 1.319 & 94.16 & 1.318 & 93.71 & 1.317 \\
\hline & & LR & 94.51 & 1.282 & 95.04 & 1.289 & 94.85 & 1.290 \\
\hline & & PB & 99.98 & 1.540 & 98.90 & 1.487 & 95.83 & 1.409 \\
\hline & & TB & 95.95 & 1.136 & 93.38 & 1.058 & 91.20 & 1.008 \\
\hline & & $\mathrm{BN}$ & 94.64 & 1.273 & 95.24 & 1.277 & 95.11 & 1.281 \\
\hline & & BA & 94.67 & 1.271 & 95.24 & 1.275 & 95.15 & 1.279 \\
\hline
\end{tabular}


In particular, the exact confidence interval (EX) is not recommended in practice, due to its computational complexity.

For interval estimation, overall, the Bayesian credible interval provides a good balance between the coverage probabilities as well as the expected widths. Therefore, we would recommend to use the Bayesian credible interval with non-informative prior in general if no prior information about the parameter is available, otherwise, the Bayesian credible interval with informative prior should be used when reliable prior information about the parameter is available. However, if one wants to guarantee the coverage probability achieves the nominal level and the width of the confidence interval and complexity of computation are not the major concerns, then the exact confidence interval (EX) should be used.

\section{Concluding Remarks}

In this paper, we proposed an adaptive Type-II progressive censoring scheme and discussed the statistical inference based on exponential lifetime data. We compared different statistical inference procedures and the performance of the MLE with the hybrid censoring scheme proposed by $[16]$.

Based on our results, the Bayesian posterior mean for point estimation and Bayesian credible interval are recommended when reliable prior information about the unknown parameter is available, otherwise, MLE for point estimation and Bayesian credible interval with noninformative prior for interval estimation should be used in general.

From this study, once again, we can see that experimenter needs to compromise between (i) minimizing the total test time; (ii) saving experimental units; and (iii) estimating efficiently, and there is always a trade-off between these three concerns. The computation formulae and results provided in this paper give a guideline on planning an experiment to compromise these three concerns. Further investigation on obtaining optimal experimental designs for given values of ideal total test time $(T)$, number of units available for test $(n)$ and the number of failures allowed for the experiment $(m)$ would be of interest in experimental planning. 


\section{Acknowledgment}

The authors thank the editor-in-chief, Professor Awi Federgruen, the past editor-in-chief, Professor Barry Nelson, the associate editor and two referees for their critical comments and helpful suggestions which led to a considerable improvement in the contents as well as the presentation of this manuscript. The authors would also like to thank Professors N. Balakrishnan and G. Iliopoulos for their suggestions. H. K. T. Ng and P. S. Chan are supported by The Research Grants Council of Hong Kong Earmarked Grant (project number 2150567). D. Kundu is supported by a grant from the Department of Science and Technology, Government of India.

\section{References}

[1] Balakrishnan, N., Progressive censoring methodology: an appraisal, Test 16 (2007), 211296 (with discussion).

[2] Balakrishnan, N. and Aggarwala, R., Progressive censoring: theory, methods and applications, Birkhäuser: Boston, MA, 2000.

[3] Balakrishnan, N. and Basu, A. P., The exponential distribution: theory, methods and applications, Gordon and Breach: Langhorne, PA, 1995.

[4] Balakrishnan, N. and Iliopoulos, G., Stochastic monotonicity of the MLE of exponential mean under different censoring schemes, to appear in Annals of the Institute of Statistical Mathematics (2009).

[5] Balakrishnan, N., Kundu, D., Ng, H. K. T. and Kannan, N., Point and interval estimation for a simple step-stress model with Type-II censoring, Journal of Quality Technology 39 (2007), 35-47.

[6] Balakrishnan, N. and Sandhu, R. A., A simple simulational algorithm for generating progressive Type-II censored samples, The American Statistician 49 (1995), 229-230. 
[7] Burkschat, M., On optimality of extremal schemes in progressive Type II censoring. Journal of Statistical Planning and Inference 138 (2008), 1647-1659.

[8] Chen, S. M. and Bhattacharya, G. K., Exact confidence bound for an exponential parameter under hybrid censoring, Communications in Statistics - Theory and Methods 16 (1988), 1857-1870.

[9] Childs, A., Chandrasekar, B., Balakrishnan, N. and Kundu, D., Exact likelihood inference based on Type-I and Type-II hybrid censored samples from the exponential distribution, Annals of the Institute of Statistical Mathematics 55 (2003), 319-330.

[10] David, H. A. and Nagaraja, H. N., Order statistics, third edition, Wiley: New York, NY, 2003.

[11] Efron, B. and Tibshirani, R., An introduction to the bootstrap, Chapman \& Hall: New York, NY, 1993.

[12] Gupta, R. D. and Kundu, D., Hybrid censoring schemes with exponential failure distribution, Communications in Statistics - Theory and Methods 27 (1998), 3065-3083.

[13] Johnson, N. L., Kotz, S. and Balakrishnan, N., Continuous univariate distributions - vol. 1, second edition, John Wiley \& Sons: New York, NY, 1994.

[14] Kamps, U. and Cramer, E., On distribution of generalized order statistics. Statistics 35 (2001), 269-280.

[15] Kundu, D. and Basu, S., Analysis of incomplete data in presence of competing risks, Journal of Statistical Planning and Inference 87 (2000), 221-239.

[16] Kundu, D. and Joarder, A., Analysis of Type-II progressively hybrid censored data, Computational Statistics and Data Analysis 50 (2006), 2509-2258.

[17] Meeker, W. Q. and Escobar, L. A., Statistical methods for reliability data, Wiley: New York, NY, 1998. 
[18] Neyman J. and Pearson E. S., On the use and interpretation of certain test criteria for purpose of statistical inference, Biometrika 20 (1928), 175-247.

[19] Ng, H. K. T. and Chan, P. S., Discussion on "Progressive censoring methodology: an appraisal” by N. Balakrishnan, Test 16 (2007), 287-289.

[20] Ng, H. K. T., Chan, P. S. and Balakrishnan, N., Optimal progressive censoring plan for the Weibull distribution, Technometrics 46 (2004), 470-481.

\section{Appendix A. Exact conditional distribution of $\hat{\lambda}$ given}

$$
J=j
$$

Consider the conditional joint cumulative distribution function of $\left(X_{1: m: n}, X_{2: m: n}, \ldots,, X_{m: m: n}\right)$ given $J=j$

$$
\begin{aligned}
& \operatorname{Pr}\left(X_{i: m: n} \leq x_{i}, i=1,2, \ldots, m \mid J=j\right) \\
& \quad=\frac{\operatorname{Pr}\left(X_{i: m: n} \leq x_{i}, i=1,2, \ldots, m, X_{j: m: n}<T \leq X_{j+1: m: n}\right)}{\operatorname{Pr}(J=j)}
\end{aligned}
$$

and differentiate both sides of the equality with respect to $x_{1}, x_{2}, \ldots, x_{m}$. We obtain the joint probability density function of $\mathbf{X}=\left(X_{1: m: n}, \ldots, X_{m: m: n}\right)$ as

$$
\begin{gathered}
f_{\mathbf{X}}\left(x_{1}, x_{2}, \ldots, x_{m} \mid J=j\right)=\frac{\left.\left[\prod_{i=1}^{m} f\left(x_{i}\right)\right]\left\{\prod_{i=1}^{j}\left[1-F\left(x_{i}\right)\right]^{R_{i}}\right\}\left[1-F\left(x_{m}\right)\right]{ }^{\left(n-m-\sum_{i=1}^{j} R_{i}\right.}\right)}{\operatorname{Pr}(J=j)}, \\
-\infty<x_{1}<\cdots<x_{j}<T<x_{j+1}<\cdots<x_{m}<\infty
\end{gathered}
$$

For exponential distribution, integrate over the region $\Omega=\left\{\left(x_{1}, \ldots, x_{m}\right) \mid x_{1}<\cdots<x_{j}<\right.$ $\left.T<x_{j+1}<\cdots<x_{m}\right\}$ and from equation (5)

$$
\int \ldots \int_{\Omega} \lambda^{m} e^{\lambda \delta(\mathbf{x}, j)} d x_{1} \ldots d x_{m}=c_{j-1} \sum_{i=1}^{j} \frac{a_{i, j}}{\left(\gamma_{i}-\gamma_{j+1}\right)}\left[e^{-\gamma_{j+1} \lambda T}-e^{-\gamma_{i} \lambda T}\right] .
$$

For notation convenience, let $\theta=1 / \lambda$ and the MLE of $\theta$ is $\hat{\theta}=\frac{\delta(\mathbf{x}, j)}{m}$. The moment generating function of $\hat{\theta}$, conditional on $J=j$ is given by

$$
E\left(e^{\omega \hat{\theta}} \mid J=j\right)=E\left(e^{\frac{\omega \delta(\mathbf{x}, j)}{m}} \mid J=j\right)
$$




$$
\begin{aligned}
& =\int \ldots \int_{\Omega} e^{\frac{\omega \delta(\mathbf{x}, j)}{m}} f_{\mathbf{X}}\left(x_{1}, \ldots, x_{m}\right) d x_{1} \ldots d x_{m} \\
& =\frac{\theta^{-m} \int \ldots \int_{\Omega} e^{-\left(\frac{1}{\theta}-\frac{\omega}{m}\right) \delta(\mathbf{x}, j)} d x_{1} \ldots d x_{m}}{\operatorname{Pr}(J=j)} \\
& =\frac{\theta^{-m}\left(\frac{m-\omega \theta}{m \theta}\right)^{-m} \int \ldots \int_{\Omega}\left(\frac{m-\omega \theta}{m \theta}\right)^{m} e^{-\left(\frac{1}{\theta}-\frac{\omega}{m}\right) \delta(\mathbf{x}, j)} d x_{1} \ldots d x_{m}}{\operatorname{Pr}(J=j)} .
\end{aligned}
$$

From equation (A.1), we can obtain

$$
\begin{aligned}
E\left(e^{\omega \hat{\theta}} \mid J=j\right) & =\frac{\left(1-\frac{\omega \theta}{m}\right)^{-m} c_{j-1} \sum_{i=1}^{j} \frac{a_{i, j}}{\left(\gamma_{i}-\gamma_{j+1}\right)}\left[e^{-\gamma_{j+1} T\left(\frac{1}{\theta}-\frac{\omega}{m}\right)}-e^{-\gamma_{i} T\left(\frac{1}{\theta}-\frac{\omega}{m}\right)}\right]}{c_{j-1} \sum_{i=1}^{j} \frac{a_{i, j}}{\left(\gamma_{i}-\gamma_{j+1}\right)}\left[e^{-\gamma_{j+1} T / \theta}-e^{-\gamma_{i} T / \theta}\right]} \\
& =\frac{\left(1-\frac{\omega \theta}{m}\right)^{-m} \sum_{i=1}^{j} \frac{a_{i, j}}{\left(\gamma_{i}-\gamma_{j+1}\right)}\left[e^{-\gamma_{j+1} T / \theta} e^{-\gamma_{j+1} T \omega / m}-e^{-\gamma_{i} T / \theta} e^{-\gamma_{i} T \omega / m}\right]}{\sum_{i=1}^{j} \frac{a_{i, j}}{\left(\gamma_{i}-\gamma_{j+1}\right)}\left[e^{-\gamma_{j+1} T / \theta}-e^{-\gamma_{i} T / \theta}\right]} .
\end{aligned}
$$

Let $Y$ be a $\operatorname{Gamma}(\alpha, \beta)$ with $\mathrm{PDF}$ defined in equation (1). For an arbitrary constant $A$, it can be shown that the moment generating function of $Y+A$ is (Johnson, Kotz and Balakrishnan [13], p. 338)

$$
M_{Y+A}(\omega)=e^{\omega A}\left(1-\frac{\omega}{\beta}\right)^{-\alpha} .
$$

It follows that the conditional $\mathrm{PDF}$ of $\hat{\theta}$, given $J=j$, is

$$
f_{\hat{\theta}}(t \mid J=j)=\frac{\sum_{i=1}^{j} \frac{a_{i, j}}{\left(\gamma_{i}-\gamma_{j+1}\right)}\left[e^{-\gamma_{j+1} T / \theta} g\left(t-\frac{\gamma_{j+1} T}{m} ; m, \frac{m}{\theta}\right)-e^{-\gamma_{i} T / \theta} g\left(t-\frac{\gamma_{i} T}{m} ; m, \frac{m}{\theta}\right)\right]}{\sum_{i=1}^{j} \frac{a_{i, j}}{\left(\gamma_{i}-\gamma_{j+1}\right)}\left[e^{-\gamma_{j+1} T / \theta}-e^{-\gamma_{i} T / \theta}\right]},
$$

$j=0,1,2, \ldots, m$. The conditional expectation and variance of the $\hat{\theta}$ are given by

$$
\begin{aligned}
E(\hat{\theta} \mid J=j) & =\theta+\frac{T A_{1}(\theta)}{m} \\
\text { and } \operatorname{Var}(\hat{\theta} \mid J=j) & =\frac{\theta^{2}}{m}+\frac{T^{2}}{m^{2}}\left[A_{2}(\theta)-A_{1}^{2}(\theta)\right],
\end{aligned}
$$

respectively, where

$$
A_{l}(\theta)=\frac{\sum_{i=1}^{j} \frac{a_{i, j}}{\left(\gamma_{i}-\gamma_{j+1}\right)}\left[\gamma_{j+1}^{l} e^{-\gamma_{j+1} T / \theta}-\gamma_{i}^{l} e^{-\gamma_{i} T / \theta}\right]}{\sum_{i=1}^{j} \frac{a_{i, j}}{\left(\gamma_{i}-\gamma_{j+1}\right)}\left[e^{-\gamma_{j+1} T / \theta}-e^{-\gamma_{i} T / \theta}\right]}, \quad l=1,2
$$


For fixed value of $j$ as the effective sample size $m$ increases, the conditional expectation of $\hat{\theta}$ converges to $\theta$ and the variance of $\hat{\theta}$ converges to 0 . Upon the transformation $\hat{\lambda}=1 / \hat{\theta}$, we have the conditional PDF of $\hat{\lambda}$, given $J=j$, as

$$
f_{\hat{\lambda}}(u \mid J=j)=\frac{\frac{1}{u^{2}} \sum_{i=1}^{j} \frac{a_{i, j}}{\left(\gamma_{i}-\gamma_{j+1}\right)}\left[e^{-\gamma_{j+1} T \lambda} g\left(\frac{1}{u}-\frac{\gamma_{j+1} T}{m} ; m, m \lambda\right)-e^{-\gamma_{i} T \lambda} g\left(\frac{1}{u}-\frac{\gamma_{i} T}{m} ; m, m \lambda\right)\right]}{\sum_{i=1}^{j} \frac{a_{i, j}}{\left(\gamma_{i}-\gamma_{j+1}\right)}\left[e^{-\gamma_{j+1} T \lambda}-e^{-\gamma_{i} T \lambda}\right]},
$$

$j=0,1,2, \ldots, m$. Note that the expectation and variance of $\hat{\lambda}$ are not in compact forms but they could be numerically obtained.

\section{Appendix B. Probability Mass Function of $J$}

For exponential distribution, the probability density function of $X_{j: m: n}$ is given by (see Balakrishnan and Aggawala [2] and Kamps and Cramer [14])

$$
f_{X_{j: m: n}}(x)=c_{j-1} \sum_{i=1}^{j} a_{i, j} \lambda \exp \left(-\gamma_{i} \lambda x\right), \quad 0<x<\infty,
$$

where $\gamma_{j}, a_{i, j}$ and $c_{j-1}$ are given in equations (2), (3) and (6), respectively.

Given $X_{j: m: n}=x_{j}, X_{j+1: m: n}$ is distributed as the first order statistic of a random sample of size $\gamma_{j+1}=n-j-\sum_{i=1}^{j} R_{i}$ from a truncated exponential distribution with CDF

$$
F_{X_{j+1: m: n}}\left(x \mid X_{j: m: n}=x_{j}\right)=1-\exp \left[-\gamma_{j+1} \lambda\left(x-x_{j}\right)\right], x_{j}<x<\infty .
$$

First, the probability that $J=0$ and $J=m$ are

$$
\begin{aligned}
\operatorname{Pr}(J=0) & =\operatorname{Pr}\left(X_{1: m: n}>T\right)=\exp (-n \lambda T), \\
\operatorname{Pr}(J=m) & =\operatorname{Pr}\left(X_{m: m: n}<T\right)=1-c_{m-1} \sum_{i=1}^{m} \frac{a_{i, m}}{\gamma_{i}} \exp \left(-r_{i} \lambda T\right),
\end{aligned}
$$

respectively.

The probability mass function of $J, J=1,2, \ldots, m-1$ is

$$
\begin{aligned}
\operatorname{Pr}(J=j) & =\operatorname{Pr}\left(X_{j: m: n}<T \leq X_{j+1: m: n}\right) \\
& =\int_{0}^{\infty} \operatorname{Pr}\left(x<T \leq X_{j+1: m: n} \mid X_{j: m: n}=x\right) f_{X_{j: m: n}}(x) d x
\end{aligned}
$$




$$
\begin{aligned}
& =\int_{0}^{T}\left[1-F_{X_{j+1: m: n}}\left(T \mid X_{j: m: n}=x_{j}\right)\right] f_{X_{j: m: n}}(x) d x \\
& =c_{j-1} \exp \left(-\gamma_{j+1} \lambda T\right) \lambda\left\{\sum_{i=1}^{j} a_{i, j} \int_{0}^{T} \exp \left[-\lambda\left(\gamma_{i}-\gamma_{j+1}\right) x\right] d x\right\} \\
& =c_{j-1} \exp \left(-\gamma_{j+1} \lambda T\right)\left\{\sum_{i=1}^{j} \frac{a_{i, j}}{\left(\gamma_{i}-\gamma_{j+1}\right)}\left[1-\exp \left(-\lambda\left(\gamma_{i}-\gamma_{j+1}\right) T\right)\right]\right\} .
\end{aligned}
$$

Therefore, we can write the PMF of $J$ as

$$
\operatorname{Pr}(J=j)=c_{j-1} \exp \left(-\gamma_{j+1} \lambda T\right) \sum_{i=1}^{j} \frac{a_{i, j}}{\left(\gamma_{i}-\gamma_{j+1}\right)}\left[1-\exp \left(-\left(\gamma_{i}-\gamma_{j+1}\right) \lambda T\right)\right]
$$

for $j=0,1, \ldots, m$, with $\gamma_{m+1} \equiv 0$ and $c_{-1} \equiv 1$. 